\title{
Optimal Consumption and Portfolio Decision with Convertible Bond in Affine Interest Rate and Heston's SV Framework
}

\author{
Hao Chang ${ }^{1,2}$ and Xue-Yan $\mathrm{Li}^{1}$ \\ ${ }^{1}$ School of Science, Tianjin Polytechnic University, Tianjin 300387, China \\ ${ }^{2}$ College of Management and Economics, Tianjin University, Tianjin 300072, China
}

Correspondence should be addressed to Hao Chang; ch8683897@126.com

Received 27 January 2016; Accepted 22 May 2016

Academic Editor: Vladimir Turetsky

Copyright ( $\odot 2016$ H. Chang and X.-Y. Li. This is an open access article distributed under the Creative Commons Attribution License, which permits unrestricted use, distribution, and reproduction in any medium, provided the original work is properly cited.

\begin{abstract}
We are concerned with an optimal investment-consumption problem with stochastic affine interest rate and stochastic volatility, in which interest rate dynamics are described by the affine interest rate model including the Cox-Ingersoll-Ross model and the Vasicek model as special cases, while stock price is driven by Heston's stochastic volatility (SV) model. Assume that the financial market consists of a risk-free asset, a zero-coupon bond (or a convertible bond), and a risky asset. By using stochastic dynamic programming principle and the technique of separation of variables, we get the HJB equation of the corresponding value function and the explicit expressions of the optimal investment-consumption strategies under power utility and logarithmic utility. Finally, we analyze the impact of market parameters on the optimal investment-consumption strategies by giving a numerical example.
\end{abstract}

\section{Introduction}

As a hot topic, investment-consumption problem has abstracted increasing attention of many investment institutions which include insurance companies, pension management institutions, and commercial banks. As a milestone of investment-consumption field, Merton [1,2] studied a continuous-time consumption and portfolio selection problem and obtained optimal investment strategies under power utility and logarithmic utility by using dynamic programming principle. Subsequently, more and more scholars paid their attentions to the investment-consumption problems. Vila and Zariphopoulou [3] researched an investmentconsumption problem with borrowing constraints. On this basis, Yao and Zhang [4] studied the investmentconsumption problem with housing risky. Duffie et al. [5] investigated the investment-consumption problem with HARA utility in incomplete markets. Dai et al. [6] investigated the investment-consumption problem with transaction costs in finite time horizon. Peng et al. [7] studied the optimal investment-consumption-proportional reinsurance problem with option type payoff. By complicated deliberation and calculation, Zhao et al. [8] got the optimal investment-consumption policies with nonexponential discounting and logarithmic utility. In order to further investigate investment-consumption problems, PalaciosHuertay and Prez-Kakabadsez [9] and de-Paz et al. [10] introduced discounting function into stochastic hyperbolic discounting function and heterogeneous discounting function, respectively. Kronborg and Steffensen [11] devoted themselves to an inconsistent investment-consumption problem and received some instructive results.

It is clear to show that the above-mentioned literatures have been achieved on the preconditions of constant interest rate and constant volatility. However, a fact has been established that some typical market parameters (such as interest rate, volatility, and inflation rate) are not invariable for a long time horizon and can be influenced by a variety of uncertain factors (e.g., disaster, war, exchange rate, and monetary policy). Hence, the introducing of stochastic interest rate or stochastic volatility makes the optimal investment strategy greatly instructive. Korn and Kraft [12] studied the portfolio selection problem with stochastic interest rate by applying stochastic control approach. Deelstra et al. [13] researched the optimal investment problem with minimum guarantee. Fleming and Pang [14] introduced stochastic control theory to investigate the consumption-investment problem with stochastic interest rate. $\mathrm{H}$. Chang and $\mathrm{K}$. 
Chang [15] used Legendre transform-dual method to study the investment-consumption problem with Vasicek model. Fleming and Hernandez-Hernandez [16] investigated the optimal consumption model with stochastic volatility. As an innovation to [16], Chacko and Viceira [17] concerned the dynamic consumption and portfolio selection problem with stochastic volatility in incomplete markets. On the latest advance of reinsurance-investment problems with the Heston model, the interested readers can refer to the works of $\mathrm{Li}$ et al. [18], Yi et al. [19], Zhao et al. [20], and A and Li [21].

Obviously, most literatures of the previous paragraph have been finished under the assumption of single uncertain factor, while real investment environment is very complicated and its interest rate and volatility should be stochastic. It is very clear that the portfolio decisions with stochastic interest rate and stochastic volatility are more practical. In recent years, some results have been obtained under the different market assumptions. For example, Liu [22] and Noh and Kim [23] investigated the optimal consumption-investment problems with stochastic interest rate and stochastic volatility, but they did not obtain the explicit solutions to the optimal consumption-investment strategies. Chang and Rong [24] studied a special optimal investment-consumption problem with CIR interest rate and stochastic volatility under the assumption that interest rate dynamics are independent of stock price dynamics. However, stock market environment in practical is greatly affected by interest rate. Guan and Liang [25] introduced stochastic interest rate and stochastic volatility into a pension management problem and received good results. Inspired by previous works, this paper considers the impact of interest rate on stock price and extends the model of Chang and Rong [24] and further studies a general investment-consumption problem with stochastic interest rate and stochastic volatility. The financial market is supposed to be composed of a risk-free asset, a zero-coupon bond, and a risky asset. In addition, short-term interest rate is assumed to be driven by affine interest rate model, and stock price is affected by interest rate dynamics and volatility dynamics simultaneously. The zero-coupon bond can be reproduced by a kind of convertible bond when there is no zero-coupon bond in the financial market. We use dynamic programming principle and the method of separation of variable to obtain the explicit solutions to the optimal investment-consumption strategies. Finally, we give a numerical example to illustrate our results.

This paper is organized as follows. In Section 2, we characterize the consumption-investment model in a stochastic affine interest rate and stochastic volatility framework. In Section 3, we use dynamic programming principle to derive the HJB equation and apply the method of separation of variable to obtain the optimal investment-consumption strategies. Numerical analysis is given in Section 4. Section 5 concludes the paper.

\section{Problem Formulation}

Let $\left(\Omega, \mathscr{F},\left\{\mathscr{F}_{t}\right\}_{t \geq 0}, P\right)$ be a filtered complete probability space; $\mathscr{F}_{t}$ represents information available at time $t$ in the market.
We assume that all processes introduced below are well defined and adapted to $\left\{\mathscr{F}_{t}\right\}_{t \geq 0}$.

2.1. Financial Market. The financial market consists of a cash, a bond, and a stock. The interest rate $r(t)$ is supposed to be a stochastic process and to be governed by

$$
\begin{aligned}
& d r(t)=(a-b r(t)) d t-\sqrt{k_{1} r(t)+k_{2}} d W_{r}(t) \\
& r(0)=r_{0},
\end{aligned}
$$

where $a, b, k_{1}$, and $k_{2}$ are positive real constants and $W_{r}(t)$ is a standard Brownian motion on $\left(\Omega, \mathscr{F},\left\{\mathscr{F}_{t}\right\}_{t \geq 0}, P\right)$. Notice that (1) consists of the Vasicek model $\left(k_{1}=0\right)$ and CIR model $\left(k_{2}=0\right)$ as special cases. In the case of $\left(k_{2}=0\right)$, the condition $2 a>b$ is required to ensure that $r(t)>0$.

The risk-free asset (i.e., cash) satisfies the following equation:

$$
\frac{d S_{0}(t)}{S_{0}(t)}=r(t) d t, \quad S_{0}(0)=S_{0} .
$$

The second asset is one zero-coupon bond with maturity $s$, whose price process $B(t, s)$ is given by the following stochastic differential equation (SDE):

$$
\begin{aligned}
\frac{d B(t, s)}{B(t, s)}= & r(t) d t+h_{0}(s-t) \sqrt{k_{1} r(t)+k_{2}} \\
& \times\left(\lambda_{r} \sqrt{k_{1} r+k_{2}} d t+d W_{r}(t)\right),
\end{aligned}
$$

where the boundary condition $B(s, s)=1$ and $\lambda_{r} \sqrt{k_{1} r(t)+k_{2}}$ is the market price of risk resulting from $W_{r}(t)$. In addition,

$$
\begin{aligned}
& h_{0}(t)=\frac{2\left(e^{m t}-1\right)}{m-\left(b-k_{1} \lambda_{r}\right)+e^{m t}\left(m+b-k_{1} \lambda_{r}\right)}, \\
& \quad m=\sqrt{\left(b-k_{1} \lambda_{r}\right)^{2}+2 k_{1}} .
\end{aligned}
$$

The maturity of the bond $B(t, T)$ is $T-t$, which varies continuously over time. Since there does not exist zerocoupon bond with any maturity $t>0$ in the market, it is unrealistic to invest in $B(t, T)$. So we introduced a convertible bond with a constant maturity $K$. We can invest in the convertible bond to hedge the risk of interest rate.

Assume that price process of the convertible bond is denoted by $B_{k}(t)$ and follows the following stochastic differential equations (SDE):

$$
\begin{aligned}
\frac{d B_{k}(t)}{B_{k}(t)}= & r(t) d t+h_{0}(K) \sqrt{k_{1} r(t)+k_{2}} \\
& \cdot\left(\lambda_{r} \sqrt{k_{1} r+k_{2}} d t+d W_{r}(t)\right) .
\end{aligned}
$$

In fact, since the convertible bond is only correlated with interest rate, it can be reproduced by the zero-coupon bond and cash:

$$
\begin{aligned}
\frac{d B(t, s)}{B(t, s)}= & \left(1-\frac{h_{0}(s-t)}{h_{0}(K)}\right) \frac{d S_{0}(t)}{S_{0}(t)}+\frac{h_{0}(s-t)}{h_{0}(K)} \\
& \cdot \frac{d B_{k}(t)}{B_{k}(t)} .
\end{aligned}
$$


The price process of risky asset (i.e., stock) is denoted by $S(t)$; then $S(t)$ satisfies the following SDE:

$$
\begin{aligned}
\frac{d S(t)}{S(t)}= & r(t) d t+\sigma_{s} \sqrt{k_{1} r(t)+k_{2}} \\
& \times\left(\lambda_{r} \sqrt{k_{1} r+k_{2}} d t+d W_{r}(t)\right)+v l(t) d t \\
& +\sqrt{l(t)} d W_{s}(t), \\
d l(t)= & \alpha(\delta-l(t)) d t+\sigma_{l} \sqrt{l(t)} d W_{l}(t),
\end{aligned}
$$

where $\alpha, \delta, \sigma_{l}$, and $v$ are positive constants. Moreover, $W_{s}(t)$ and $W_{l}(t)$ are standard Brownian motion with $\operatorname{Cov}(W(t), W(t))=\rho_{s l} t$, and $W_{r}(t)$ is independent of $W_{s}(t)$ and $W_{l}(t)$; the price of risk of Brownian motion $W_{s}(t)$ is given by $v \sqrt{l(t)}$.

2.2. Wealth Process. During the time horizon $[0, T]$, we assume that the initial wealth of an investor is given by $X_{0}>$ 0 and the wealth value is $X(t)$ at time $t$. We denote the consumption amount, the proportion of the wealth invested in the convertible bond, and the stock at time $t$ by $\widetilde{C}(t), \pi_{B}(t)$, and $\pi_{s}(t)$, respectively. In addition, $\pi_{0}(t)$ is the proportion of wealth invested in the cash, so $\pi_{0}(t)=1-\pi_{B}(t)-$ $\pi_{s}(t)$. Therefore, wealth process $X(t)$ evolves according to the following equation:

$$
\begin{aligned}
d X(t)= & \pi_{0}(t) X(t) \frac{d S_{0}(t)}{S_{0}(t)}+\pi_{B}(t) X(t) \frac{d B_{k}(t)}{B_{k}(t)} \\
& +\pi_{s}(t) X(t) \frac{d S(t)}{S(t)}-\widetilde{C}(t) d t, \quad X(0)=X_{0} .
\end{aligned}
$$

Substituting (2), (5), and (7) into (9), we can get

$$
\begin{aligned}
& d X(t)=[r(t) X(t) \\
& \quad+u_{B}(t) h_{0}(K) \lambda_{r} X(t)\left(k_{1} r(t)+k_{2}\right) \\
& \quad+\pi_{s}(t) \sigma_{s} \lambda_{r}\left(k_{1} r(t)+k_{2}\right) X(t)+v \pi_{s}(t) l(t) X(t) \\
& \quad-\widetilde{C}(t)] d t+\left[X(t) \sqrt{k_{1} r(t)+k_{2}} \pi_{B}(t) h_{0}(K)\right. \\
& \left.\quad+X(t) \sqrt{k_{1} r(t)+k_{2}} \pi_{s}(t) \sigma_{s}\right] d W_{r}(t) \\
& \quad+X(t) \pi_{s}(t) \sqrt{l(t)} d W_{s}(t), \quad X(0)=X_{0}>0 .
\end{aligned}
$$

Definition 1. The investment-consumption strategy $\left(\pi_{B}(t)\right.$, $\left.\pi_{s}(t), \widetilde{C}(t)\right)$ is said to be admissible if the following conditions are satisfied:

(i) $\left(\pi_{B}(t), \pi_{s}(t), \widetilde{C}(t)\right)$ is $\mathscr{F}_{t}$-progressively measurable on $\left(\Omega, \mathscr{F}_{,},\left\{\mathscr{F}_{t}\right\}_{t \geq 0}, P\right)$.

(ii) $\mathbb{E}\left(\int_{0}^{T} X(t)^{2}\left[\left(\pi_{B}(t) h_{0}(k)+\pi_{S}(t) \sigma_{s}\right)^{2}\left(k_{1} r(t)+k_{2}\right)+\right.\right.$ $\left.\left.\pi_{s}(t)^{2} l(t)\right] d t\right)<\infty$.

(iii) Equation (10) has a unique strong solution for any $\left(\pi_{B}(t), \pi_{s}(t), \widetilde{C}(t)\right)$.
2.3. Objective Function. Assume that the set of all admissible strategies could be denoted by $\Gamma=\left\{\left(\widetilde{C}(t), \pi_{B}(t), \pi_{s}(t)\right): t \in\right.$ $[0, T]\}$. Investors wish to maximize the expected discounted utility of the intermediate consumption and terminal wealth; namely, our optimization problem can be formulated as follows:

$$
\begin{aligned}
& \sup _{\left(\pi_{s}(t), \pi_{B}(t), \widetilde{C}(t)\right) \in \Gamma} \mathbb{E}\left[\theta \int_{0}^{T} e^{-\beta t} U_{1}(\widetilde{C}(t)) d t\right. \\
& \left.+(1-\theta) e^{-\beta T} U_{2}(X(T))\right],
\end{aligned}
$$

where $U_{1}(\cdot)$ and $U_{2}(\cdot)$ are utility functions which are strictly concave. $\beta$ is the subject discount rate and $\theta$ determines the relative importance of the intermediate consumption and the bequest. When $\theta=0$, the expected utility only depends on the terminal wealth and problem (11) is reduced to an asset allocation problem.

\section{Optimal Consumption and Portfolio Decision}

In this section, we obtain the HJB equation of the value function by using dynamic programming principle. We define the value function as

$$
\begin{aligned}
& H(t, r, l, x) \\
& \quad=\sup _{\left(\pi_{s}(t), \pi_{B}(t), \widetilde{C}(t)\right) \in \Gamma} \mathbb{E}\left[\theta \int_{0}^{T} e^{-\beta t} U_{1}(\widetilde{C}(t)) d t\right. \\
& +(1-\theta) e^{-\beta T} U_{2}(X(T)) \mid X(t)=x, r(t)=r, l(t) \\
& \quad=l]
\end{aligned}
$$

with the boundary condition $H(T, r, l, x)=(1-\theta) e^{-\beta T} U_{2}(x)$.

Using dynamic programming principle, we can get the corresponding HJB equation:

$$
\begin{aligned}
& \sup _{\left(\pi_{s}(t), \pi_{B}(t), \widetilde{C}(t)\right) \in \Gamma}\left\{H_{t}+(a-b r) H_{r}+\frac{1}{2}\left(k_{1} r+k_{2}\right) H_{r r}\right. \\
& +(\delta-l) \alpha H_{l}+\frac{1}{2} \sigma_{l}^{2} l H_{l l}+[r x \\
& +x\left(\pi_{B}(t) h_{0}(K)+\pi_{s} \sigma_{s}\right)\left(\lambda_{r}\left(k_{1} r+k_{2}\right)\right)+x \pi_{s} v l \\
& -\widetilde{C}(t)] H_{x}+\frac{1}{2} x^{2}\left[\left(\pi_{B}(t) h_{0}(K)+\pi_{s}(t) \sigma_{s}\right)^{2}\right. \\
& \left.\times\left(k_{1} r+k_{2}\right)+\pi_{s}(t)^{2} l\right] H_{x x}+\rho_{s l} \sigma_{l} x l \pi_{s}(t) H_{l x} \\
& -\left[x\left(\pi_{B}(t) h_{0}(K)+\pi_{s}(t) \sigma_{s}\right)\left(k_{1} r+k_{2}\right)\right] H_{x r} \\
& \left.+\theta e^{-\beta t} U_{1}(\widetilde{C}(t))\right\}=0,
\end{aligned}
$$

where $H_{t}, H_{r}, H_{r r}, H_{l}, H_{l l}, H_{x}, H_{x x}, H_{x r}$, and $H_{l x}$ are the first-order, second-order, and mixed partial derivatives of the 
value function with respect to the variables $t, r, l$, and $x$. We use similar symbol to represent partial derivatives of the other functions in latter part of this paper.

Using first-order maximizing conditions for the optimal investment and consumption strategies, we get

$$
\begin{aligned}
\pi_{s}^{*}(t)= & \frac{-v H_{x}}{x H_{x x}}-\frac{\rho_{s l} \sigma_{l} H_{l x}}{x H_{x x}}, \\
\pi_{B}^{*}(t)= & \frac{H_{x r}}{h_{0}(K) x H_{x x}}+\frac{v \sigma_{s}-\lambda_{r}}{x h_{0}(K)} \frac{H_{x}}{H_{x x}} \\
& +\frac{\rho_{s l} \sigma_{l} \sigma_{s} H_{l x}}{x h_{0}(K) H_{x x}}, \\
\dot{U}_{1}\left(\widetilde{C}^{*}(t)\right)= & \frac{H_{x}}{\theta e^{-\beta t}} .
\end{aligned}
$$

Putting (14) into (13), we obtain the HJB equation as follows:

$$
\begin{aligned}
H_{t}+ & (r x-\widetilde{C}(t)) H_{x}+(a-b r) H_{r} \\
+ & \frac{1}{2}\left(k_{1} r+k_{2}\right) H_{r r}+\alpha(\delta-l) H_{l} \\
& -\frac{1}{2}\left(\lambda_{r}^{2}\left(k_{1} r+k_{2}\right)+v^{2} l\right) \frac{H_{x}^{2}}{H_{x x}}-\rho_{s l} \sigma_{l} v l \frac{H_{x} H_{l x}}{H_{x x}} \\
& +\lambda_{r}\left(k_{1} r+k_{2}\right) \frac{H_{x} H_{x r}}{H_{x x}}-\frac{\left(k_{1} r+k_{2}\right) H_{x r}^{2}}{2 H_{x x}} \\
& -\frac{l \rho_{s l}^{2} \sigma_{l}^{2}}{2} \frac{H_{l x}^{2}}{H_{x x}}+\frac{1}{2} \sigma_{l}^{2} l H_{l l}+\theta e^{-\beta t} U_{1}(\widetilde{C}(t))=0 .
\end{aligned}
$$

In this paper, we assume that the risk aversion degree of investors can be described by power utility and logarithmic utility, respectively. We use variable change technique to investigate the optimal investment-consumption strategies under power utility and logarithmic utility.

3.1. Power Utility. Power utility is given by $U_{1}(x)=U_{2}(x)=$ $x^{\gamma} / \gamma, \gamma<1$ and $\gamma \neq 0$, where $\gamma$ is the risk aversion factor. We conjecture the solution to problem (15) with the following form:

$$
H(t, r, l, x)=e^{-\beta t} \frac{x^{\gamma}}{\gamma} f(t, r, l), \quad f(T, r, l)=1-\theta .
$$

The partial derivatives of (16) are as follows:

$$
\begin{aligned}
H_{t} & =\frac{x^{\gamma}}{\gamma} e^{-\beta t}\left(-\beta f+f_{t}\right), \\
H_{x} & =e^{-\beta t} x^{\gamma-1} f, \\
H_{x x} & =(\gamma-1) e^{-\beta t} x^{\gamma-2} f, \\
H_{r} & =\frac{x^{\gamma}}{\gamma} e^{-\beta t} f_{r}, \\
H_{r r} & =\frac{x^{\gamma}}{\gamma} e^{-\beta t} f_{r r},
\end{aligned}
$$

$$
\begin{aligned}
H_{l} & =\frac{x^{\gamma}}{\gamma} e^{-\beta t} f_{l}, \\
H_{l l} & =\frac{x^{\gamma}}{\gamma} e^{-\beta t} f_{l l}, \\
H_{x r} & =e^{-\beta t} x^{\gamma-1} f_{r}, \\
H_{x l} & =e^{-\beta t} x^{\gamma-1} f_{l} .
\end{aligned}
$$

Therefore, we get the optimal consumption policy as follows:

$$
\widetilde{C}(t)=\left(\frac{f}{\theta}\right)^{1 /(\gamma-1)} x .
$$

Substituting (17) and (18) back into (15), we derive

$$
\begin{aligned}
& \frac{x^{\gamma}}{\gamma} e^{-\beta t}\left\{f_{t}\right. \\
& +\left[r \gamma-\beta-\frac{1}{2}\left(\lambda_{r}^{2} \frac{\gamma}{\gamma-1}\left(k_{1} r+k_{2}\right)+v^{2} l\right)\right] f \\
& +\left[\lambda_{r} \frac{\gamma}{\gamma-1}\left(k_{1} r+k_{2}\right)+a-b r\right] f_{r} \\
& +\left(\alpha(\delta-l)+\frac{\gamma}{\gamma-1} \rho_{s l} \sigma_{l} v l\right) f_{l}+\frac{1}{2} \sigma_{l}^{2} l f_{l l} \\
& -\frac{l}{2} \frac{\gamma}{\gamma-1} \rho_{s l}^{2} \sigma_{l}^{2} \frac{f_{l}^{2}}{f}-\frac{\gamma}{\gamma-1} \frac{\left(k_{1} r+k_{2}\right) f_{r}^{2}}{2 f} \\
& \left.+\frac{k_{1} r+k_{2}}{2} f_{r r}(1-\gamma) \theta^{1 /(1-\gamma)} f^{\gamma /(1-\gamma)}\right\}=0 .
\end{aligned}
$$

Eliminating the dependence on $x$, we obtain

$$
\begin{aligned}
f_{t}+\left[r \gamma-\beta-\frac{1}{2}\left(\lambda_{r}^{2}\left(k_{1} r+k_{2}\right)+v^{2} l\right) \frac{\gamma}{\gamma-1}\right] f \\
+\left[\lambda_{r} \frac{\gamma}{\gamma-1}\left(k_{1} r+k_{2}\right)+a-b r\right] f_{r} \\
+\left(\alpha(\delta-l)+\frac{\gamma}{\gamma-1} \rho_{s l} \sigma_{l} v l\right) f_{l}+\frac{1}{2} \sigma_{l}^{2} l f_{l l} \\
\quad-\frac{l}{2} \frac{\gamma}{\gamma-1} \rho_{s l}^{2} \sigma_{l}^{2} \frac{f_{l}^{2}}{f}-\frac{\gamma}{\gamma-1} \frac{\left(k_{1} r+k_{2}\right) f_{r}^{2}}{2 f} \\
+\frac{k_{1} r+k_{2}}{2} f_{r r}+(1-\gamma) \theta^{1 /(1-\gamma)} f^{\gamma /(\gamma-1)}=0 .
\end{aligned}
$$

Assume that the solution to (20) is given by

$$
f(t, r, l)=u(t, r, l)^{1-\gamma}, \quad u(T, r, l)=(1-\theta)^{1 /(1-\gamma)} .
$$


The partial derivatives of the above function are as follows:

$$
\begin{aligned}
& f_{t}=(1-\gamma) u^{-\gamma} u_{t}, \\
& f_{r}=(1-\gamma) u^{-\gamma} u_{r}, \\
& f_{r r}=(1-\gamma) u^{-\gamma}\left(-\gamma \frac{u_{r}^{2}}{u}+u_{r r}\right), \\
& f_{l}=(1-\gamma) u^{-\gamma} u_{l}, \\
& f_{l l}=(1-\gamma) u^{-\gamma}\left(-\gamma \frac{u_{l}^{2}}{u}+u_{l l}\right) .
\end{aligned}
$$

Putting (22) into (20), we can get

$$
\begin{aligned}
& (1-\gamma) u^{-\gamma}\left[u_{t}+\left[\lambda_{r} \frac{\gamma}{\gamma-1}\left(k_{1} r+k_{2}\right)+(a-b r)\right] u_{r}\right. \\
& -\frac{1}{2}\left(\rho_{s l}^{2}-1\right) \sigma_{l}^{2} \gamma l \frac{u_{l}^{2}}{u}+\left(\frac{r \gamma}{1-\gamma}-\frac{\beta}{1-\gamma}\right. \\
& \left.-\frac{\gamma}{(\gamma-1)^{2}} \cdot \frac{\lambda_{r}^{2}\left(k_{1} r+k_{2}\right)+v^{2} l}{2}\right) u+[\alpha(\delta-l) \\
& \left.-\frac{\gamma}{\gamma-1} \rho_{s l} \sigma_{l} v l\right] u_{l}+\frac{1}{2}\left(k_{1} r+k_{2}\right) u_{r r}+\frac{1}{2} \sigma_{l}^{2} l u_{l l} \\
& \left.+\theta^{1 /(1-\gamma)}\right]=0 .
\end{aligned}
$$

Due to $(1-\gamma) u^{-\gamma} \neq 0$, we can get the partial differential equation as follows:

$$
\begin{aligned}
u_{t}+ & \frac{1}{1-\gamma}\left(r \gamma-\beta+\frac{\gamma}{\gamma-1} \frac{\lambda_{r}^{2}\left(k_{1} r+k_{2}\right)+v^{2} l}{2}\right) u \\
& +\left[\alpha(\delta-l)-\frac{\gamma}{\gamma-1} \rho_{s l} \sigma_{l} v l\right] u_{l} \\
& -\frac{1}{2} \gamma \sigma_{l}^{2}\left(\rho_{s l}^{2}-1\right) l \frac{u_{l}^{2}}{u} \frac{1}{2}\left(k_{1} r+k_{2}\right) u_{r r}+\frac{1}{2} \sigma_{l}^{2} l u_{l l} \\
& +\theta^{1 /(1-\gamma)}=0, \quad u(T, r, l)=(1-\theta)^{1 /(1-\gamma)}
\end{aligned}
$$

For (24), we find that this equation is similar to (22) in the paper of Chang and Rong [24]. Inspired by the method of Chang and Rong [24], we give Lemma 2.

Lemma 2. Suppose that

$$
\begin{aligned}
u(t, r, l)= & \theta^{1 /(1-\gamma)} \int_{t}^{T} \widetilde{u}(s, r, l) d s \\
& +(1-\theta)^{1 /(1-\gamma)} \widetilde{u}(t, r, l)
\end{aligned}
$$

is the solution to (24); then $\tilde{u}(t, r, l)$ satisfies

$$
\begin{aligned}
\tilde{u}_{t}+ & \frac{1}{1-\gamma}\left(r \gamma-\beta+\frac{\gamma}{\gamma-1} \frac{\lambda_{r}^{2}\left(k_{1} r+k_{2}\right)+v^{2} l}{2}\right) \tilde{u} \\
+ & {\left[-\frac{\gamma}{\gamma-1} \rho_{s l} \sigma_{l} v l+\alpha(\delta-l)\right] \tilde{u}_{l} } \\
& -\frac{1}{2}\left(\rho_{s l}^{2}-1\right) \gamma \sigma_{l}^{2} l \frac{\tilde{u}_{l}^{2}}{\widetilde{u}}+\frac{1}{2}\left(k_{1} r+k_{2}\right) \tilde{u}_{r r} \\
& +\frac{1}{2} \sigma_{l}^{2} l \tilde{u}_{l l}=0, \quad \tilde{u}(T, r, l)=1 .
\end{aligned}
$$

Proof. For any function $u(t, r, l)$, we define the following variational operator:

$$
\begin{aligned}
& \nabla u(t, r, l) \\
& =\frac{1}{1-\gamma}\left(r \gamma-\beta+\frac{\gamma}{1-\gamma} \cdot \frac{\lambda_{r}^{2}\left(k_{1} r+k_{2}\right)+l v^{2}}{2}\right) u \\
& +\left[\alpha(\delta-l)-\frac{\gamma}{\gamma-1} \rho_{s l} \sigma_{l} v l\right] u_{l} \\
& \quad-\frac{1}{2}\left(\rho_{s l}^{2}-1\right) \gamma \sigma_{l}^{2} l \frac{u_{l}^{2}}{u}+\frac{1}{2}\left(k_{1} r+k_{2}\right) u_{r r} \\
& \quad+\frac{1}{2} \sigma_{l}^{2} l u_{l l} .
\end{aligned}
$$

Then we can rewrite (24) as

$$
\frac{\partial u}{\partial t}+\nabla u+\theta^{1 /(1-\gamma)}=0, \quad u(T, r, l)=(1-\theta)^{1 /(1-\gamma)} .
$$

In addition, we get

$$
\begin{aligned}
\frac{\partial u(t, r, l)}{\partial t}= & -\theta^{1 /(1-\gamma)} \widetilde{u}(t, r, l) \\
& +(1-\theta)^{1 /(1-\gamma)} \frac{\partial \widetilde{u}(t, r, l)}{\partial t} \\
= & \theta^{1 /(1-\gamma)}\left(\int_{t}^{T} \frac{\partial \widetilde{u}(s, r, l)}{\partial s} d s-\widetilde{u}(T, r, l)\right) \\
& +(1-\theta)^{1 /(1-\gamma)} \frac{\partial \widetilde{u}(t, r, l)}{\partial t}, \\
\nabla u(t, r, l)= & \theta^{1 /(1-\gamma)} \int_{t}^{T} \nabla \widetilde{u}(s, r, l) d s \\
& +(1-\theta)^{1 /(1-\gamma)} \nabla \widetilde{u}(t, r, l) .
\end{aligned}
$$

Putting (29) in (28), we derive

$$
\begin{aligned}
& \theta^{1 /(1-\gamma)}\left(\int_{t}^{T}\left(\frac{\partial \widetilde{u}(s, r, l)}{\partial s}+\nabla \widetilde{u}(s, r, l)\right) d s-\widetilde{u}(T, r, l)\right. \\
& +1)+(1-\theta)^{1 /(1-\gamma)}\left(\frac{\partial \widetilde{u}(t, r, l)}{\partial t}+\nabla \widetilde{u}(t, r, l)\right) \\
& \quad=0 .
\end{aligned}
$$


So we have

$$
\frac{\partial \widetilde{u}(t, r, l)}{\partial t}+\nabla \widetilde{u}(t, r, l)=0, \quad \widetilde{u}(T, r, l)=1 .
$$

Namely, (26) holds. It is obvious that we can verify (26).

Lemma 3. Assume that the solution to (26) is given by

$$
\tilde{u}(t, r, l)=\exp \{A(t)+B(t) r+D(t) l\}
$$

with the boundary condition $A(T)=B(T)=D(T)=0$; then $A(t), B(t)$, and $D(t)$ are given by (52), (45), and (50), respectively.

Proof. The partial derivatives of (32) are as follows:

$$
\begin{aligned}
\widetilde{u}_{t} & =(\dot{A}(t)+\dot{B}(t) r+\dot{D}(t) l) \tilde{u}, \\
\widetilde{u}_{r} & =B(t) \widetilde{u}, \\
\widetilde{u}_{r r} & =B^{2}(t) \widetilde{u}, \\
\widetilde{u}_{l} & =D(t) \widetilde{u}, \\
\widetilde{u}_{l l} & =D^{2}(t) \tilde{u} .
\end{aligned}
$$

Plugging (33) into (26), we can get

$$
\begin{aligned}
& {\left[\dot{B}(t)+\frac{k_{1}}{2} B^{2}(t)+\left(\lambda_{r} k_{1} \frac{\gamma}{1-\gamma}-b\right) B(t)+\frac{\gamma}{1-\gamma}\right.} \\
& \left.+\frac{\lambda_{r}^{2} k_{1} \gamma}{2(\gamma-1)^{2}}\right] r+[\dot{D}(t) \\
& \quad+\frac{1}{2} \sigma_{l}^{2}\left(\gamma\left(\rho_{s l}^{2}-1\right)+1\right) D^{2}(t) \\
& \left.-\left(\rho_{s l} \sigma_{l} v \frac{\gamma}{\gamma-1}+\alpha\right) D(t)+\frac{v^{2} \gamma}{2(1-\gamma)^{2}}\right] l[\dot{A}(t) \\
& +\frac{k_{2}}{2} B^{2}(t)+\left(\frac{\gamma}{\gamma-1} \lambda_{r} k_{2}+a\right) B(t)+\alpha \delta D(t) \\
& \left.-\frac{\beta}{1-\gamma}-\frac{\lambda_{r}^{2} k_{2} \gamma}{2(1-\gamma)^{2}}\right]=0 .
\end{aligned}
$$

Eliminating the dependence on $r$ and $l$, we obtain the following three equations:

$$
\begin{aligned}
\dot{B}(t) & +\frac{k_{1}}{2} B^{2}(t)+\left(\frac{\gamma}{1-\gamma} \lambda_{r} k_{1}-b\right) B(t)+\frac{\gamma}{1-\gamma} \\
& +\frac{\lambda_{r}^{2} k_{1} \gamma}{2(\gamma-1)^{2}}=0, \\
\dot{D}(t) & +\frac{1}{2} \sigma_{l}^{2}\left(\gamma\left(\rho_{s l}^{2}-1\right)+1\right) D^{2}(t) \\
& -\left(\frac{\gamma}{\gamma-1} \rho_{s l} \sigma_{l} v \alpha\right) D(t)+\frac{v^{2} \gamma}{2(1-\gamma)^{2}}=0,
\end{aligned}
$$

$$
\begin{aligned}
\dot{A}(t) & +\frac{k_{2}}{2} B^{2}(t)+\left(\frac{\gamma}{\gamma-1} \lambda_{r} k_{2}+a\right) B(t)+\alpha \delta D(t) \\
& -\frac{\beta}{1-\gamma}+\frac{\lambda_{r}^{2} k_{2} \gamma}{2(1-\gamma)^{2}}=0 .
\end{aligned}
$$

Equation (35) can be written as follows:

$$
\begin{aligned}
\dot{B}(t)= & -\frac{k_{1}}{2} B^{2}(t)+\left(b-\frac{\gamma}{1-\gamma} \lambda_{r} k_{1}\right) B(t)-\frac{\gamma}{1-\gamma} \\
& -\frac{\lambda_{r}^{2} k_{1} \gamma}{2(\gamma-1)^{2}} .
\end{aligned}
$$

The discriminant of quadratic equation

$$
\begin{gathered}
-\frac{k_{1}}{2} B^{2}(t)+\left(b-\frac{\gamma}{1-\gamma} \lambda_{r} k_{1}\right) B(t)-\frac{\gamma}{1-\gamma} \\
-\frac{\lambda_{r}^{2} k_{1} \gamma}{2(\gamma-1)^{2}}=0
\end{gathered}
$$

is denoted by $\Delta_{B}$. Then

$$
\Delta_{B}=\left(b-\frac{\gamma}{\gamma-1} \lambda_{r} k_{1}\right)^{2}-\frac{k_{1}^{2} \lambda_{r}^{2} \gamma}{(\gamma-1)^{2}}-\frac{2 k_{1} \gamma}{1-\gamma} .
$$

Suppose that $\Delta_{B}>0$; we can get

$$
\gamma<\frac{b^{2}}{\left(k_{1} \lambda_{r}-b\right)^{2}+2 k_{1}} .
$$

Combining with the premise condition $\gamma<1$ and $\gamma \neq 0$, we get

$$
\gamma<\min \left(\frac{b^{2}}{\left(k_{1} \lambda_{r}-b\right)^{2}+2 k_{1}}, 1\right), \quad \gamma \neq 0 .
$$

Doing an integral calculation for (38), we obtain

$$
\begin{aligned}
& \frac{1}{\lambda_{1}-\lambda_{2}} \int_{t}^{T}\left(\frac{1}{B(t)-\lambda_{1}}-\frac{1}{B(t)-\lambda_{2}}\right) d t \\
& =-\frac{k_{1}}{2}(T-t),
\end{aligned}
$$

where $\lambda_{1}$ and $\lambda_{2}$ are two different roots of the above quadratic equation and $\lambda_{1}$ and $\lambda_{2}$ are given by

$$
\lambda_{1,2}=\frac{-(\gamma /(\gamma-1)) \lambda_{r} k_{1}+b}{k_{1}} \mp \frac{\sqrt{\Delta_{B}}}{k_{1}} .
$$

Under the boundary condition $B(T)=0$, the solution to (35) is as follows:

$$
B(t)=\frac{\lambda_{1} \lambda_{2}\left[1-\exp \left(\left(-k_{1} / 2\right)(T-t)\left(\lambda_{1}-\lambda_{2}\right)\right)\right]}{\lambda_{1}-\lambda_{2} \exp \left(\left(-k_{1} / 2\right)(T-t)\left(\lambda_{1}-\lambda_{2}\right)\right)} .
$$


Using the same method as (35), we rewrite (36) in the following form:

$$
\begin{aligned}
\dot{D}(t)= & -\frac{1}{2} \sigma_{l}^{2}\left(\gamma\left(\rho_{s l}^{2}-1\right)+1\right) D^{2}(t) \\
& +\left(\frac{\gamma}{\gamma-1} \rho_{s l} \sigma_{l} v+\alpha\right) D(t)-\frac{v^{2} \gamma}{2(1-\gamma)^{2}} .
\end{aligned}
$$

The discriminant of quadratic equation

$$
\begin{aligned}
& -\frac{1}{2} \sigma_{l}^{2}\left(\gamma\left(\rho_{s l}^{2}-1\right)+1\right) D^{2}(t) \\
& \quad+\left(\frac{\gamma}{\gamma-1} \rho_{s l} \sigma_{l} v+\alpha\right) D(t)-\frac{v^{2} \gamma}{2(1-\gamma)^{2}}=0
\end{aligned}
$$

is denoted by $\Delta_{D}$. Then

$$
\begin{aligned}
\Delta_{D}= & \left(\alpha+\frac{\gamma}{\gamma-1} \rho_{s l} \sigma_{l} v\right)^{2} \\
& -\frac{v^{2} \sigma_{l}^{2} \gamma}{(1-\gamma)^{2}}\left(\left(\rho_{s l}^{2}-1\right) \gamma+1\right) .
\end{aligned}
$$

Suppose that $\Delta_{D}>0$, and then we can get $\gamma<\alpha^{2} /\left(\left(v \sigma_{l}+\right.\right.$ $\left.\left.\alpha \rho_{s l}\right)^{2}+\alpha^{2}\left(\rho_{s l}^{2}-1\right)\right)$. We can easily see that $\alpha^{2} /\left(\left(v \sigma_{l}+\alpha \rho_{s l}\right)^{2}+\right.$ $\left.\alpha^{2}\left(\rho_{s l}^{2}-1\right)\right)<1$. Combining with the premise conditions $\gamma<1$ and $\gamma \neq 0$, we get

$$
\gamma<\frac{\alpha^{2}}{\left(v \sigma_{l}+\alpha \rho_{s l}\right)^{2}+\alpha^{2}\left(\rho_{s l}^{2}-1\right)}, \quad \gamma \neq 0 .
$$

Using the same calculation as (35), we obtain

$$
D(t)=\frac{\lambda_{3} \lambda_{4}\left(1-c_{0}\right)}{\lambda_{3}-\lambda_{4} c_{0}}
$$

where $\lambda_{3,4}=\left(\alpha+(\gamma /(\gamma-1)) \rho_{s l} \sigma_{l} v\right) / \sigma_{l}^{2}\left(1+\gamma\left(\sigma_{s l}^{2}-1\right)\right) \mp$ $\sqrt{\Delta_{C}} / \sigma_{l}^{2}\left(1+\gamma\left(\sigma_{s l}^{2}-1\right)\right)$ and $c_{0}=\exp \left\{-(1 / 2) \sigma_{l}^{2}\left(1-\gamma\left(\rho_{s l}^{2}-\right.\right.\right.$ 1)) $\left.\left(\lambda_{3}-\lambda_{4}\right)(T-t)\right\}$.

Combining (42) and (49), we can get

$$
\begin{aligned}
\gamma<\min \left\{\frac{\alpha^{2}}{\left(v \sigma_{l}+\alpha \rho_{s l}\right)^{2}+\alpha^{2}\left(\rho_{s l}^{2}-1\right)},\right. \\
\left.\frac{b^{2}}{\left(k_{1} \lambda_{r}-b\right)^{2}+2 k_{1}}\right\} .
\end{aligned}
$$

Solving (37), we get

$$
\begin{aligned}
A(t)= & \frac{k_{2}}{2} \int_{t}^{T} B^{2}(s) d s \\
& +\left(\frac{\gamma}{\gamma-1} \lambda_{r} k_{2}+a\right) \int_{t}^{T} B(s) d s \\
& +\alpha \delta \int_{t}^{T} D(s) d s+\left(\frac{\lambda_{r}^{2} k_{2} \gamma}{2(1-\gamma)^{2}}-\frac{\beta}{1-\gamma}\right) t .
\end{aligned}
$$

So Lemma 3 is proved.
To sum up, we have the following conclusion.

Theorem 4. If utility function is given by $U_{1}(x)=U_{2}(x)=$ $x^{\gamma} / \gamma, \gamma<1$ and $\gamma \neq 0$, then, under the condition of $\gamma<$ $\min \left\{\alpha^{2} /\left(\left(v \sigma_{l}+\alpha \rho_{s l}\right)^{2}+\alpha^{2}\left(\rho_{s l}^{2}-1\right)\right), b^{2} /\left(\left(k_{1} \lambda_{r}-b\right)^{2}+2 k_{1}\right)\right\}$, the optimal consumption-investment strategies for problem (11) are given by

$$
\begin{aligned}
\pi_{s}^{*}(t)= & \frac{v}{(1-\gamma)}+\rho_{s l} \sigma_{l} D(t), \\
\pi_{B}^{*}(t)= & -\frac{1}{h_{0}(K)} B(t)+\frac{v \sigma_{s}-\lambda_{r}}{h_{0}(K)(\gamma-1)} \\
& -\frac{\rho_{s l} \sigma_{l} \sigma_{s}}{h_{0}(K)} D(t), \\
\widetilde{C}^{*}(t)= & \frac{\theta^{1 /(1-\gamma)}}{u(t, r, l)} X(t),
\end{aligned}
$$

where $h_{0}(K), B(t)$, and $D(t)$ are given by (4), (45), and (50), respectively.

3.2. Logarithmic Utility. Logarithmic utility is given by

$$
U_{1}(x)=U_{2}(x)=\ln (x)
$$

We conjecture the solution to problem (15) with the following form:

$$
\begin{aligned}
& H(t, r, l, x)=g(t) e^{-\beta t} \ln x+h(t, r, l), \\
& \qquad g(T)=1-\theta, h(T, r, l)=0 .
\end{aligned}
$$

Then partial derivatives are as follows:

$$
\begin{aligned}
H_{t} & =\ln x\left(\dot{g}(t) e^{-\beta t}-\beta g(t) e^{-\beta t}\right)+h_{t}, \\
H_{x} & =\frac{g(t) e^{-\beta t}}{x}, \\
H_{x x} & =\frac{g(t) e^{-\beta t}}{-x^{2}}, \\
H_{r} & =h_{r}, \\
H_{r r} & =h_{r r} \\
H_{l} & =h_{l}, \\
H_{l l} & =h_{l l}, \\
H_{x r} & =0 \\
H_{x l} & =0 .
\end{aligned}
$$


Substituting (56) into (15), we get

$$
\begin{aligned}
\ln x( & \left.\dot{g}(t) e^{-\beta t}-\beta g(t) e^{-\beta t}\right)+h_{t}+r g(t) e^{-\beta t} \\
+ & \frac{1}{2}\left[\lambda_{r}^{2}\left(k_{1} r+k_{2}\right)+l v^{2}\right] g(t) e^{-\beta t}+(a-b r) h_{r} \\
& +\frac{1}{2}\left(k_{1} r+k_{2}\right) h_{r r}+\alpha(\delta-l) h_{l}+\frac{1}{2} \sigma_{l}^{2} l h_{l l} \\
& -\widetilde{C}(t) \frac{g(t) e^{-\beta t}}{x}+\theta e^{-\beta t} U_{1}(\widetilde{C}(t))=0 .
\end{aligned}
$$

Under the logarithmic utility, we have the consumption strategy with the following form:

$$
\widetilde{C}(t)=\frac{\theta}{g(t)} x .
$$

Substituting (58) into (57), we obtain

$$
\begin{aligned}
e^{-\beta t} \ln x[\dot{g}(t)-\beta g(t)+\theta]+h_{t}+(a-b r) h_{r} \\
+\frac{1}{2}\left(k_{1} r+k_{2}\right) h_{r r}+\alpha(\delta-l) h_{l}+\frac{1}{2} \sigma_{l}^{2} l h_{l l} \\
+\left(r+\frac{1}{2} \lambda_{r}^{2}\left(k_{1} r+k_{2}\right)+\frac{l v^{2}}{2}\right) g(t) e^{-\beta t} \\
+\theta e^{-\beta t}\left(\ln \frac{\theta}{g(t)}-1\right)=0 .
\end{aligned}
$$

Eliminating the dependence on $x$, we obtain

$$
\begin{aligned}
& \dot{g}(t)-\beta g(t)+\theta=0 \\
& h_{t}+(a-b r) h_{r}+\frac{1}{2}\left(k_{1} r+k_{2}\right) h_{r r}+\alpha(\delta-l) h_{l} \\
& \quad+\frac{1}{2} \sigma_{l}^{2} l h_{l l}+\left(r+\frac{1}{2} \lambda_{r}^{2}\left(k_{1} r+k_{2}\right)+\frac{l v^{2}}{2}\right) g(t) e^{-\beta t} \\
& \quad+\theta e^{-\beta t}\left(\ln \left(\frac{\theta}{g(t)}\right)-1\right)=0 .
\end{aligned}
$$

Applying the boundary condition $g(T)=1-\theta$, we get

$$
g(t)=(1-\theta) e^{-\beta(T-t)}-\frac{\theta}{\beta}\left(e^{-\beta(T-t)}-1\right) .
$$

Lemma 5. Assume that the solution to (61) is given by $h(t, r, l)=D_{1}(t)+E(t) r+F(t) l$, with boundary conditions given by $D_{1}(T)=0, E(T)=0$ and $F(T)=0$; then $D_{1}(t), E(t)$, and $F(t)$ are given by (69), (67), and (68), respectively.

Proof. The partial derivatives are as follows:

$$
\begin{aligned}
h_{t} & =\dot{D}_{1}(t)+\dot{E}(t) r+\dot{F}(t) l, \\
h_{r} & =E(t), \\
h_{r r} & =0 \\
h_{l} & =F(t), \\
h_{l l} & =0 .
\end{aligned}
$$

Putting (62) into (61), we obtain

$$
\begin{aligned}
\dot{D}_{1}(t) & +\dot{E}(t) r+\dot{F}(t) l \\
& +\left(r+\frac{1}{2} \lambda_{r}^{2}\left(k_{1} r+k_{2}\right)+\frac{l v^{2}}{2}\right) g(t) e^{-\beta t} \\
& +(a-b r) E(t)+\alpha(\delta-l) F(t) \\
& +\theta e^{-\beta t}\left(\ln \left(\frac{\theta}{g(t)}\right)-1\right)=0 .
\end{aligned}
$$

Doing a further reduction for (64), we obtain

$$
\begin{aligned}
r(\dot{E}( & \left.t)+g(t) e^{-\beta t}+\frac{1}{2} \lambda_{r}^{2} k_{1} r g(t) e^{-\beta t}-b E(t)\right) \\
& +l\left(\dot{F}(t)+\frac{v^{2}}{2} g(t) e^{-\beta t}-\alpha F(t)\right)+\dot{D}_{1}(t) \\
& +\frac{1}{2} \lambda_{r}^{2} k_{2} g(t) e^{-\beta t}+a E(t)+\alpha \delta F(t) \\
& +\theta e^{-\beta t}\left(\ln \left(\frac{\theta}{g(t)}\right)-1\right)=0 .
\end{aligned}
$$

Eliminating the dependence on $r$ and $l$, we obtain

$$
\begin{aligned}
& \dot{E}(t)+g(t) e^{-\beta t}+\frac{1}{2} \lambda_{r}^{2} k_{1} r g(t) e^{-\beta t}-b E(t)=0, \\
& \dot{F}(t)+\frac{v^{2}}{2} g(t) e^{-\beta t}-\alpha F(t)=0, \\
& \dot{D}_{1}(t)+a E(t)+\alpha \delta F(t)+\frac{1}{2} \lambda_{r}^{2} k_{2} g(t) e^{-\beta t} \\
& +\theta e^{-\beta t}\left(\ln \left(\frac{\theta}{g(t)}\right)-1\right)=0 .
\end{aligned}
$$

Under the boundary condition $D_{1}(T)=E(T)=F(T)=$ 0 , we get

$$
\begin{gathered}
E(t)=\left(\frac{1}{2} \lambda_{r}^{2} k_{1}+1\right) \\
\cdot\left(\frac{\theta \beta+\theta-\beta}{\theta \beta} e^{-\beta T}\left(e^{-b(T-t)}-1\right)\right)-\left(\frac{1}{2} \lambda_{r}^{2} k_{1}+1\right) \\
\cdot \frac{\theta}{(\theta+\beta) \beta} e^{-\beta T}\left(e^{-b(T-t)}-e^{-\beta(T-t)}\right), \\
F(t)=\frac{v^{2}}{2} \frac{\beta \theta+\theta-\beta}{\theta \beta} e^{-\beta T}\left(e^{-\alpha(T-t)}-1\right)-\frac{v^{2}}{2} \\
\cdot \frac{\theta}{(\theta+\beta) \beta} e^{-\beta T}\left(e^{-\alpha(T-t)}-e^{-\beta(T-t)}\right)
\end{gathered}
$$




$$
\begin{aligned}
& D_{1}(t)=a \int_{t}^{T} E(s) d s+\alpha \delta \int_{t}^{T} F(s) d s-\frac{1}{2} \\
& \cdot \lambda_{r}^{2} k_{2} \int_{t}^{T} g(s) e^{-\beta s} d s-\theta \int_{t}^{T} e^{-\beta s} \ln g(s) d s \\
& \quad+\theta(\ln \theta-1) \int_{t}^{T} e^{-\beta s} d s .
\end{aligned}
$$

Therefore, Lemma 5 holds.

Putting (56) into (14), we can get the following conclusion.

Theorem 6. Under logarithmic utility $U_{1}(x)=U_{2}(x)=$ $\ln (x)$, the optimal consumption-investment strategies for problem (11) are given by

$$
\begin{aligned}
& \pi_{s}^{*}(t)=v, \\
& \pi_{B}^{*}(t)=\frac{\lambda_{r}-v \sigma_{s}}{h_{0}(K)} \\
& \widetilde{C}^{*}(t)=\frac{\theta}{(1-\theta) e^{-\beta(T-t)}-(\theta / \beta)\left(e^{-\beta(T-t)}-1\right)} X(t) .
\end{aligned}
$$

\section{Numerical Example}

In this section, we give a numerical example to illustrate the optimal investment-consumption strategy under power utility. In order to analyze the impact of the parameters on the optimal strategies, we assume that the main parameters are given by $a=0.18, b=0.23, k_{1}=0.07, k_{2}=0.09, r_{0}=0.05$, $\lambda_{r}=1, \sigma_{s}=0.02, v=1.5, l_{0}=0.02, \alpha=0.03, \delta=0.04$, $\sigma_{l}=0.03, \theta=0.4, \beta=0.1, \gamma=-2, t=0, K=1, T=2$, $x_{0}=100$, and $\rho_{s l}=0.5$.

From the strategy expressions above, we can see that the trend of strategy can be influenced by a lot of market parameters, such as interest rate, volatility, discount rate, and risk aversion factor. In this section, we will analyze the sensitivity of the optimal investment-consumption strategies to the parameters $b, k_{1}, \alpha$, and $\gamma$, respectively.

As Figure 1 shows, the wealth proportion invested in stock, bond, and cash displays different trends with the increasing of $b$. The dotted line means that $b$ has no effect on $\pi_{s}^{*}(t)$. In addition, the astroid line shows that $\pi_{B}^{*}(t)$ has a decreasing trend and the broken line shows that $\pi_{0}^{*}(t)$ has an increasing trend. From (1), we see that a larger $b$ leads to a smaller expectation of interest rate. Therefore, the proportion invested in convertible bond will decrease. From the views of practical investment, this conclusion is consistent with our intuition.

From Figure 2, we see a similar trend as Figure 1. That is to say, investors will reduce the wealth proportion invested in bond and increase the wealth proportion invested in cash with the growth of $k_{1}$. As a matter of fact, an increasing $k_{1}$ means a decreasing volatility of stock price, which displays that the volatility of convertible bond is increasing. So, investors can reduce the proportion in the convertible bond in order to avoid the risk of investment. However, we find that the optimal amount in the stock remains fixed, although $k_{1}$

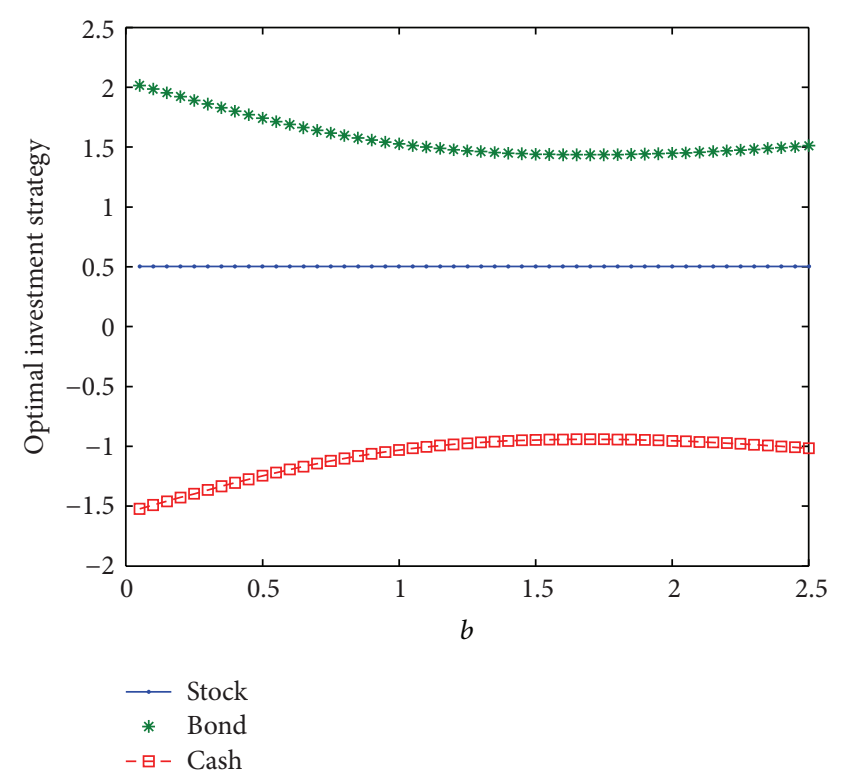

FIGURE 1: The effect of $b$ on the optimal investment strategy.

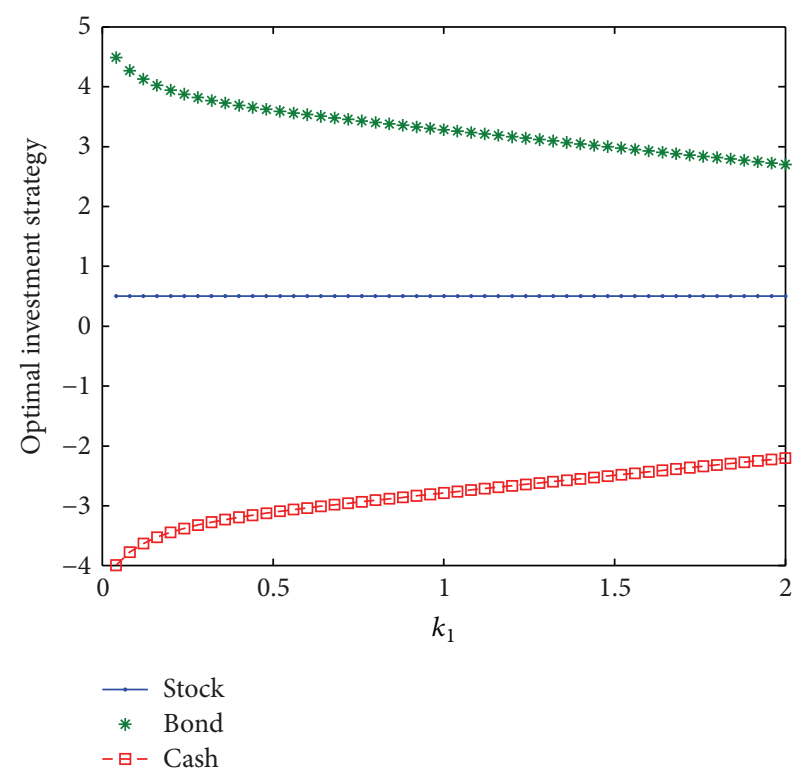

FIGURE 2: The effect of $k_{1}$ on the optimal investment strategy.

has an effect on the dynamics of stock price from (7). This conclusion surprised us. We should keep it in mind in the practical investments.

Figure 3 shows us that $\pi_{s}^{*}(t)$ increases with respect to the parameter $\alpha$, while $\pi_{B}^{*}(t)$ remains fixed value and $\pi_{0}^{*}(t)$ decreases with respect to the parameter $\alpha$. From (8), we can see that the expectation value of volatility will decrease when the value of $\alpha$ is increasing. It means that the risks from stochastic volatility will descend. Therefore, the decreasing risks lead to the increasing wealth proportion invested in the stock. This conclusion conforms to the economic implication of $\alpha$. 


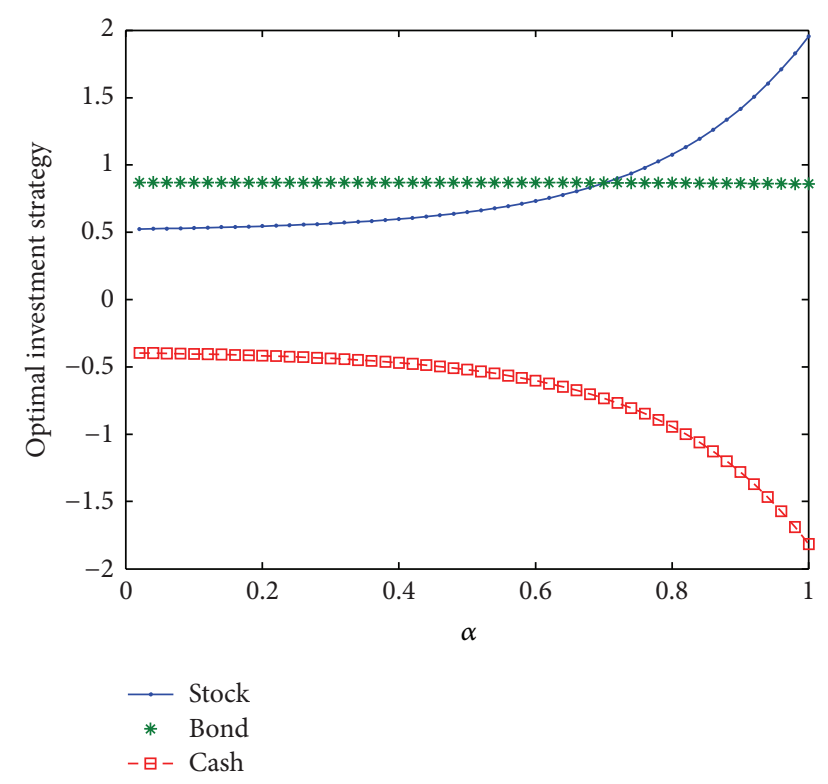

FIGURE 3: The effect of $\alpha$ on the optimal investment strategy.

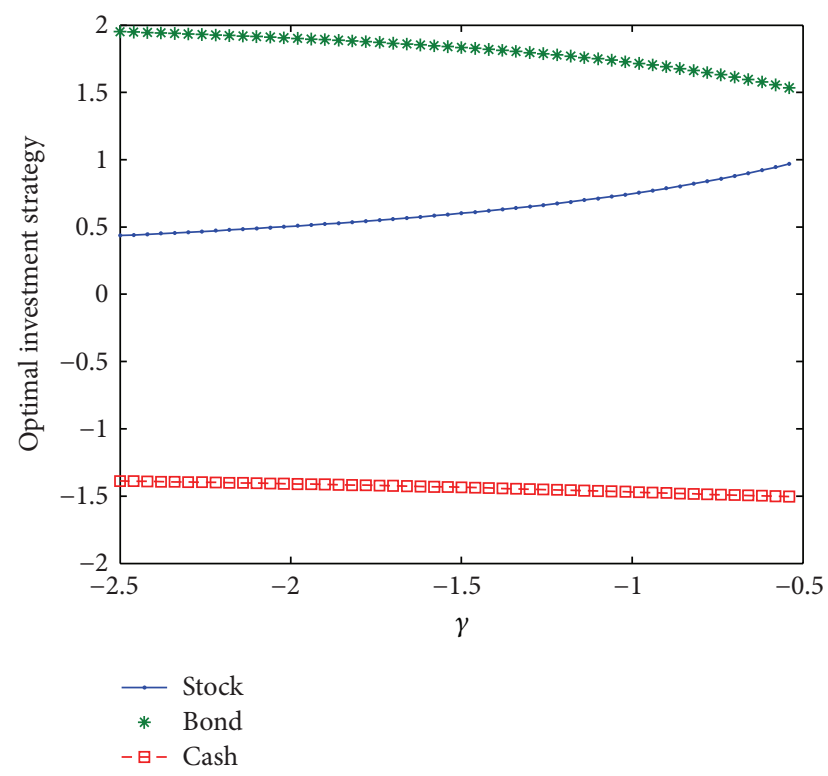

FIGURE 4: The effect of $\gamma$ on the optimal investment strategy.

Figure 4 tells us that $\pi_{s}^{*}(t)$ increases in $\gamma$, while $\pi_{B}^{*}(t)$ and $\pi_{0}^{*}(t)$ decrease in $\gamma$. From the views of utility theory, the risk aversion coefficient for power utility is given by $1-$ $\gamma$. This means that the degree of risk aversion of investors will descend when the value of $\gamma$ is ascending. Therefore, investors would invest more money in the stock and reduce the proportion of wealth in cash and convertible bond. This conclusion agrees with that obtained by Guan and Liang [25].

In Figures 5-8, $y$-axis represents the optimal consumption rate.

As seen in Figure 5, the optimal consumption rate increases with the increasing of the parameter $b$.

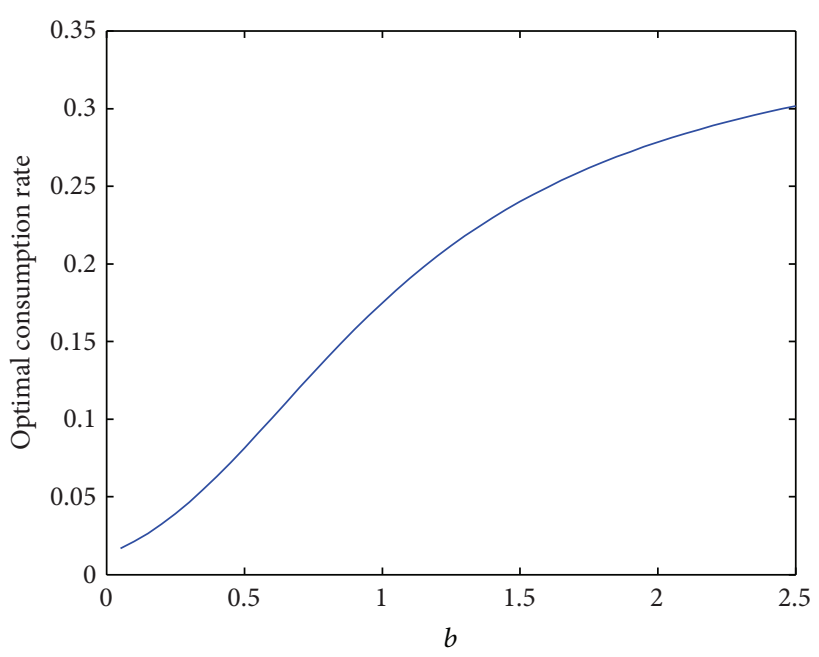

Figure 5: The effect of $b$ on the optimal consumption rate.

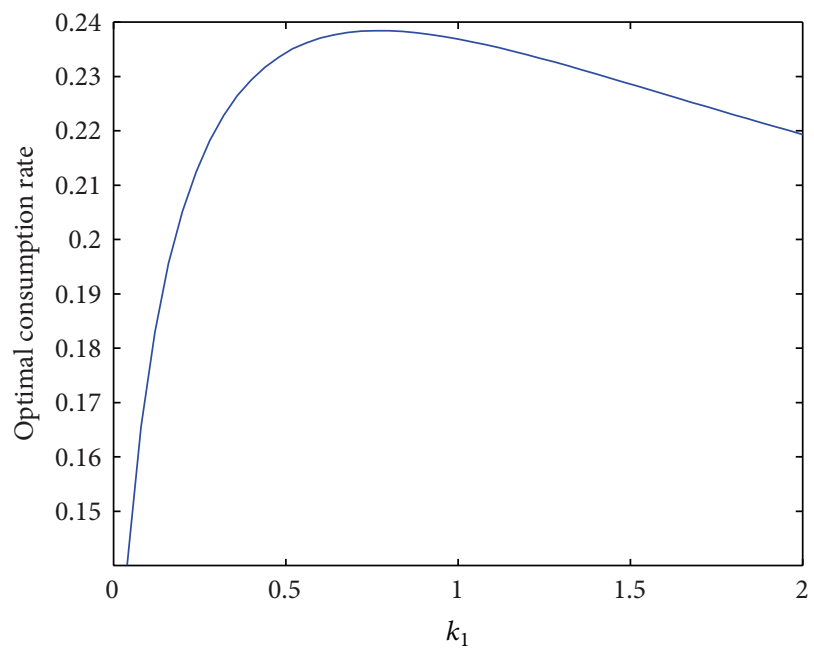

FIgURE 6: The effect of $k_{1}$ on the optimal consumption rate.

Figure 6 describes the effect of $k_{1}$ on the optimal consumption rate. We can see that there is a positive correlation between $k_{1}$ and the optimal consumption rate when $k_{1} \leq$ 0.75 , while there exists a negative correlation when $k_{1}>0.75$.

In contrast to Figure 5, Figure 7 shows that the optimal consumption rate will decrease with the growth of $\alpha$. It tells us that investors would cut down consumption expenses as $\alpha$ gets larger.

From Figure 8, we can learn the impact of $\gamma$ on the optimal consumption rate. Moreover, we obtain a strictly monotonic increasing function in Figure 8. It can be seen that the wealth of investors will also increase when the value of $\gamma$ is increasing. It means that investors may possess more wealth to consume. Therefore, the consumption rate of investors will increase accordingly as $\gamma$ is rising. This conclusion is consistent with that in the paper of Chang and Rong [24]. 


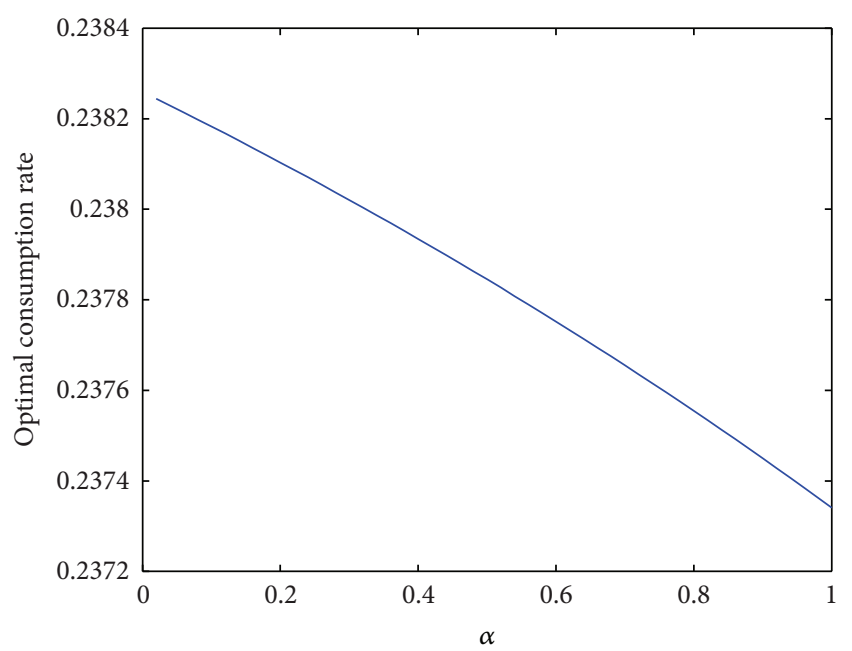

Figure 7: The effect of $\alpha$ on the optimal consumption rate.

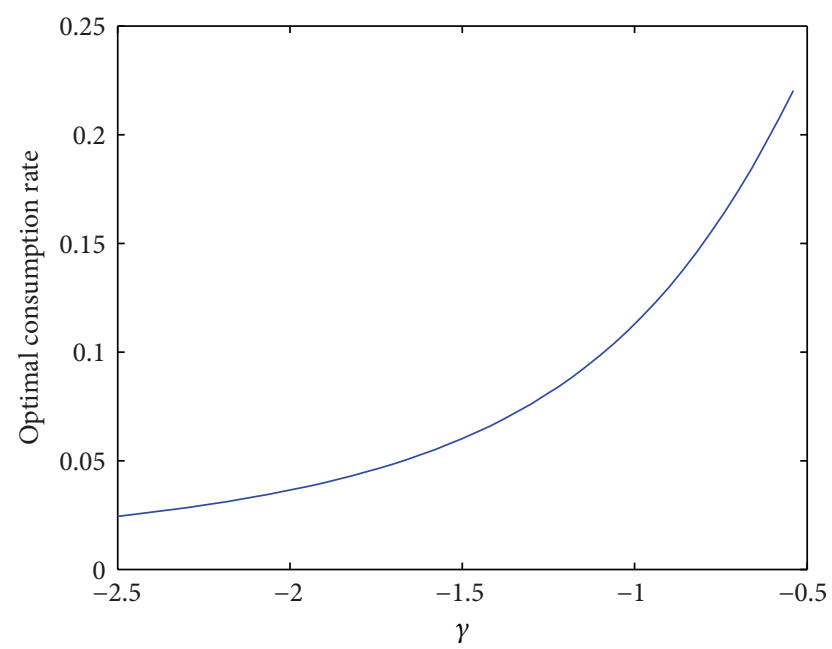

Figure 8: The effect of $\gamma$ on the optimal consumption rate.

\section{Conclusions}

In this paper, we studied optimal consumption and portfolio decision with affine interest rate and stochastic volatility. To hedge interest rate risk, we introduced a zero-coupon bond into financial market. In addition, the financial market is composed of a risk-free asset, a risky asset, and a zero-coupon bond, which can be reproduced by convertible bond. We assume that interest rate follows an affine interest rate model, while stock price is influenced by interest rate dynamics and volatility dynamics. The objective of this paper is to maximize the expected discount utility of intermediate consumption and terminal wealth. By using dynamic programming principle and method of separation of variable, we obtain the explicit expressions of the optimal investment-consumption strategy under power utility and logarithmic utility. Finally, we give a numerical example to illustrate the impact of market parameters on the optimal investment-consumption strategy and analyze economic implications of market parameters.

\section{Competing Interests}

The authors declare that there are no competing interests regarding the publication of this paper.

\section{Acknowledgments}

This research is supported by China Postdoctoral Science Foundation Funded Project (no. 2014M560185 and no. 2016T90203) and Tianjin Natural Science Foundation of China (no. 15JCQNJC04000).

\section{References}

[1] R. C. Merton, "Lifetime portfolio selection under uncertainty: the continuous-time case," The Review of Economics and Statistics, vol. 51, no. 3, pp. 247-257, 1969.

[2] R. C. Merton, "Optimum consumption and portfolio rules in a continuous-time model," Journal of Economic Theory, vol. 3, no. 4, pp. 373-413, 1971.

[3] J.-L. Vila and T. Zariphopoulou, "Optimal consumption and portfolio choice with borrowing constraints," Journal of Economic Theory, vol. 77, no. 2, pp. 402-431, 1997.

[4] R. Yao and H. H. Zhang, "Optimal consumption and portfolio choices with risky housing and borrowing constraints," The Review of Financial Studies, vol. 18, no. 1, pp. 197-239, 2005.

[5] D. Duffie, W. Fleming, H. M. Soner, and T. Zariphopoulou, "Hedging in incomplete markets with HARA utility," Journal of Economic Dynamics and Control, vol. 21, no. 4-5, pp. 753-782, 1997.

[6] M. Dai, L. Jiang, P. Li, and F. Yi, "Finite horizon optimal investment and consumption with transaction costs," SIAM Journal on Control and Optimization, vol. 48, no. 2, pp. 11341154, 2009.

[7] X. C. Peng, L. X. Wei, and Y. J. Hu, "Optimal investment, consumption and proportional reinsurance for an insurer with option type payoff," Insurance: Mathematics and Economics, vol. 59, pp. 78-86, 2014.

[8] Q. Zhao, Y. Shen, and J. Q. Wei, "Consumption-investment strategies with non-exponential discounting and logarithmic utility," European Journal of Operational Research, vol. 238, no. 3, pp. 824-835, 2014.

[9] I. Palacios-Huertay and A. Prez-Kakabadsez, "Consumption and portfolio rules with stochastic hyperbolic discounting," Akerlanak, vol. 13, no. 72, 2013.

[10] A. de-Paz, J. Marłn-Solano, J. Navas, and O. Roch, “Consumption, investment and life insurance strategies with heterogeneous discounting," Insurance: Mathematics and Economics, vol. 54, pp. 66-75, 2014.

[11] M. T. Kronborg and M. Steffensen, "Inconsistent investment and consumption problems," Applied Mathematics and Optimization, vol. 71, no. 3, pp. 473-515, 2015.

[12] R. Korn and H. Kraft, "A stochastic control approach to portfolio problems with stochastic interest rates," SIAM Journal on Control and Optimization, vol. 40, no. 4, pp. 1250-1269, 2001.

[13] G. Deelstra, M. Grasselli, and P.-F. Koehl, "Optimal investment strategies in the presence of a minimum guarantee," Insurance: Mathematics and Economics, vol. 33, no. 1, pp. 189-207, 2003.

[14] W. H. Fleming and T. Pang, "An application of stochastic control theory to financial economics," SIAM Journal on Control and Optimization, vol. 43, no. 2, pp. 502-531, 2004. 
[15] H. Chang and K. Chang, "Legendre transform-dual solution for investment and consumption problem under the Vasicek model," Journal of Systems Science and Complexity, vol. 27, no. 5, pp. 911-927, 2014.

[16] W. H. Fleming and D. Hernandez-Hernandez, "An optimal consumption model with stochastic volatility," Finance and Stochastics, vol. 7, no. 2, pp. 245-262, 2003.

[17] G. Chacko and L. M. Viceira, "Dynamic consumption and portfolio choice with stochastic volatility in incomplete markets," The Review of Financial Studies, vol. 18, no. 4, pp. 1369-1402, 2005.

[18] Z. Li, Y. Zeng, and Y. Lai, "Optimal time-consistent investment and reinsurance strategies for insurers under Heston's SV model," Insurance: Mathematics and Economics, vol. 51, no. 1, pp. 191-203, 2012.

[19] B. Yi, Z. F. Li, F. G. Viens, and Y. Zeng, "Robust optimal control for an insurer with reinsurance and investment under Heston's stochastic volatility model," Insurance: Mathematics and Economics, vol. 53, no. 3, pp. 601-614, 2013.

[20] H. Zhao, X. M. Rong, and Y. G. Zhao, "Optimal excess-of-loss reinsurance and investment problem for an insurer with jumpdiffusion risk process under the Heston model," Insurance: Mathematics and Economics, vol. 53, no. 3, pp. 504-514, 2013.

[21] C. A and Z. F. Li, "Optimal investment and excess-of-loss reinsurance problem with delay for an insurer under Heston's SV model," Insurance: Mathematics and Economics, vol. 61, pp. 181196, 2015.

[22] J. Liu, "Portfolio selection in stochastic environments," Review of Financial Studies, vol. 20, no. 1, pp. 1-39, 2007.

[23] E.-J. Noh and J.-H. Kim, "An optimal portfolio model with stochastic volatility and stochastic interest rate," Journal of Mathematical Analysis and Applications, vol. 375, no. 2, pp. 510$522,2011$.

[24] H. Chang and X.-M. Rong, "An investment and consumption problem with CIR interest rate and stochastic volatility," Abstract and Applied Analysis, vol. 2013, Article ID 219397, 12 pages, 2013.

[25] G. H. Guan and Z. X. Liang, "Optimal management of DC pension plan in a stochastic interest rate and stochastic volatility framework," Insurance: Mathematics and Economics, vol. 57, pp. 58-66, 2014. 


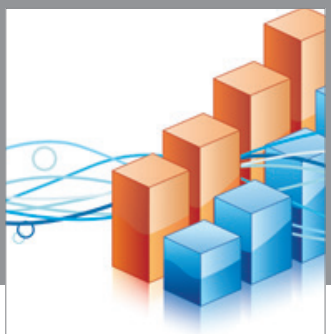

Advances in

Operations Research

vatem alat4

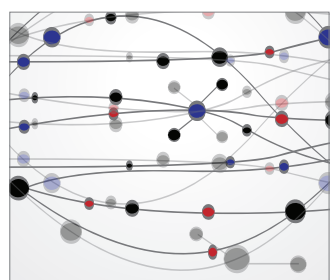

\section{The Scientific} World Journal
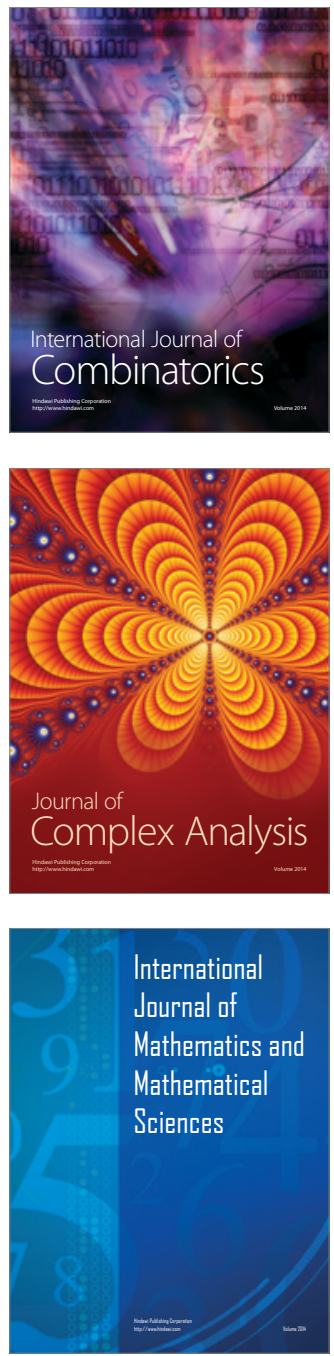
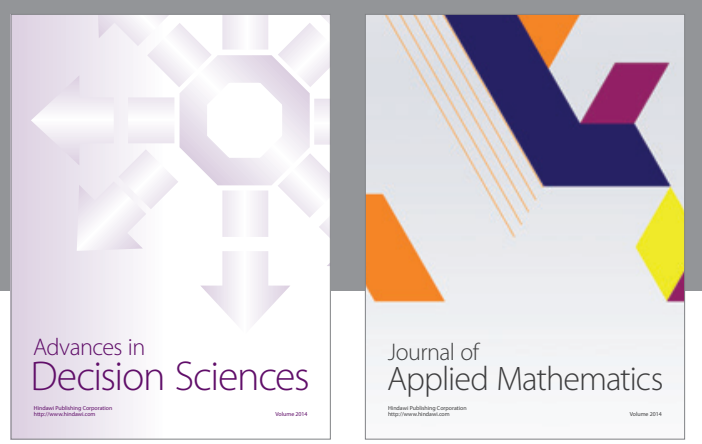

Algebra

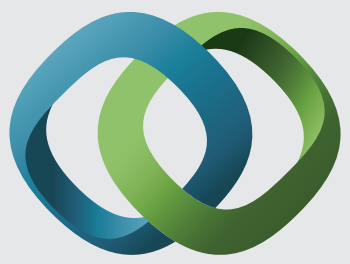

\section{Hindawi}

Submit your manuscripts at

http://www.hindawi.com
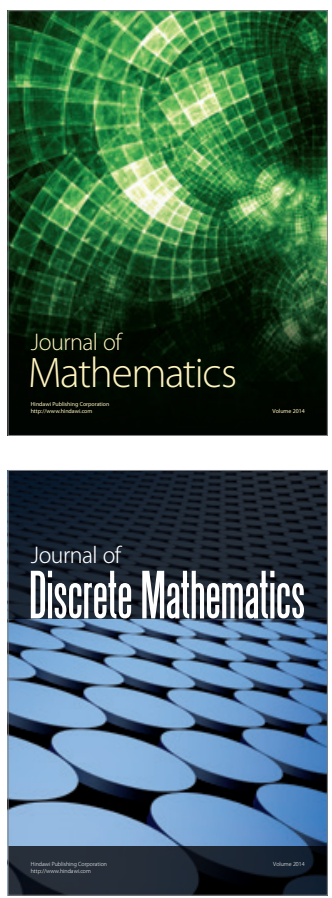

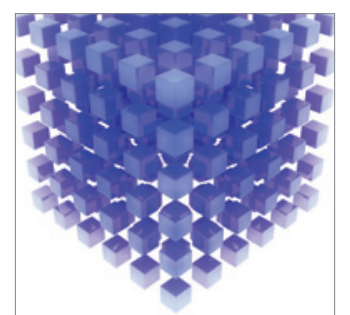

Mathematical Problems in Engineering
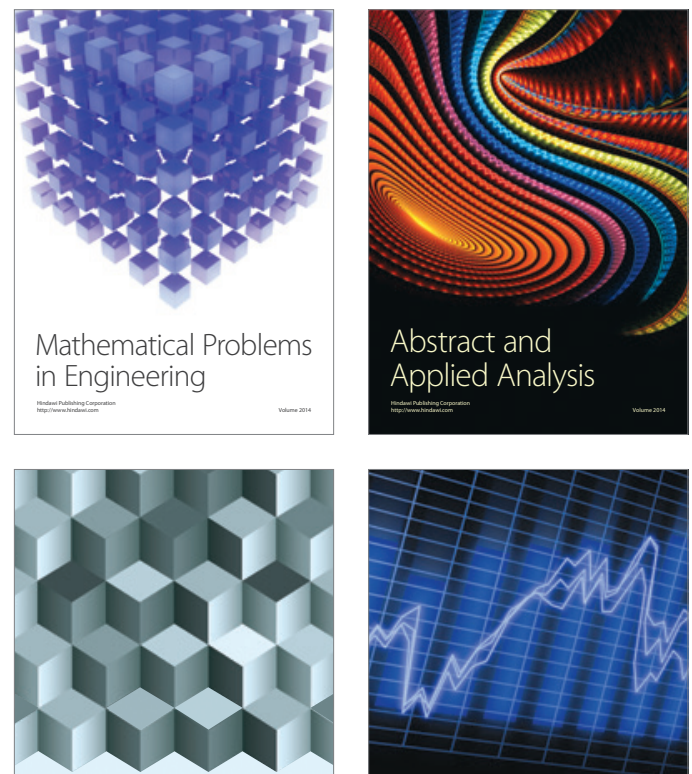

Journal of

Function Spaces

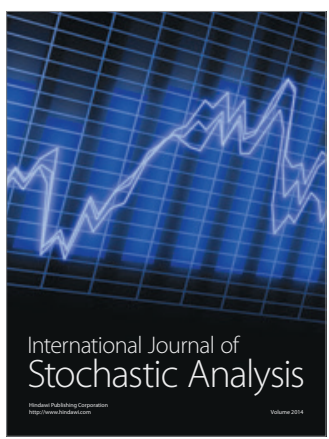

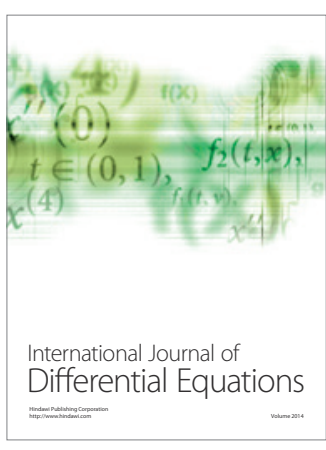
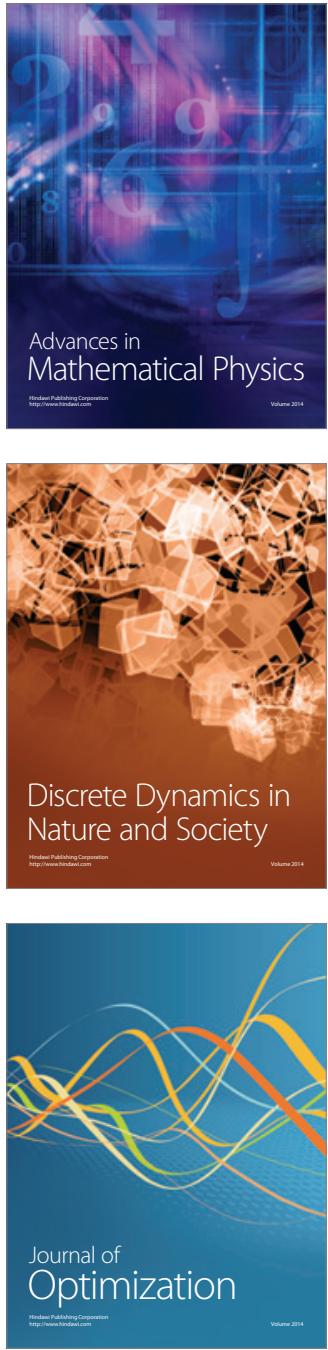